\title{
Muscimol Acts in Dorsomedial but Not Paraventricular Hypothalamic Nucleus to Suppress Cardiovascular Effects of Stress
}

\author{
Elizabeth H. Stotz-Potter, Lynn R. Willis, and Joseph A. DiMicco \\ Department of Pharmacology and Toxicology, Indiana University School of Medicine, Indianapolis, Indiana 46202
}

Both the dorsomedial hypothalamic nucleus $(\mathrm{DMH})$ and the paraventricular hypothalamic nucleus (PVN) have been implicated in the neural control of the cardiovascular response to stress. We used the $\mathrm{GABA}_{\mathrm{A}}$ agonist muscimol to inhibit neuronal activation and attempted to identify hypothalamic nuclei required for the cardiovascular response to air stress. Chronically instrumented rats received bilateral injections of either $80 \mathrm{pmol}$ of muscimol or $100 \mathrm{nl}$ of saline vehicle into the $\mathrm{DMH}$, the PVN, or an intermediate area (including the rostral edge of the $\mathrm{DMH}$ and the region between the two nuclei) and were placed immediately in a restraining tube and subjected to $20 \mathrm{~min}$ of air stress. In all rats, air stress after vehicle injections caused marked increases in heart rate (137 \pm 6 beats $/ \mathrm{min}$ ) and blood pressure $(26 \pm 2 \mathrm{mmHg})$. Microinjection of muscimol into the DMH suppressed the heart rate and blood pressure response by 85 and $68 \%$, respectively. Identical microinjection of muscimol into the intermediate area between the DMH and the PVN attenuated the increases in heart rate by only $46 \%$ and in blood pressure by $52 \%$. In contrast, similar injections into the vicinity of the PVN failed to alter the cardiovascular response to air stress. These findings demonstrate that muscimol-induced inhibition of neuronal activity in the region of the $\mathrm{DMH}$ blocks air stressinduced increases in heart rate and arterial pressure, whereas similar treatment in the area of the PVN has no effect.

Key words: dorsomedial hypothalamic nucleus; paraventricular nucleus; muscimol; GABA; heart rate; blood pressure; stress; rats
Stress is thought to contribute to various human cardiovascular disorders, including hypertension, cardiac arrhythmia, myocardial infarction, and sudden death. An area that has been accorded a primary role among higher brain centers involved in the integration of the cardiovascular response to stress is the hypothalamus. Recently, chemical stimulation of the paraventricular hypothalamic nucleus (PVN) has been reported to elicit tachycardia, increases in blood pressure (Gelsema et al., 1989; Jin and Rockhold, 1989; Martin et al., 1991; Martin and Haywood, 1993), and hemodynamic changes (Martin et al., 1993) resembling those observed in acute experimental stress in rats. Furthermore, electrolytic lesions of this region in the same species abolish the cardiovascular response to footshock stress (Callahan et al., 1989). These data are in keeping with the long-standing suggestion of a central role for the PVN in the integrated neuroendocrine and autonomic responses to stress (Swanson and Sawchenko, 1983).

Recent findings, however, have implicated a second hypothalamic region, the dorsomedial hypothatamic nucleus (DMH), in stress-induced cardiovascular changes. Microinjection of the $\mathrm{GABA}_{\mathrm{A}}$ receptor antagonist bicuculline methiodide (BMI) or of excitatory amino acids into the DMH clicits cardiovascular effects and other physiological and behavioral changes similar to those observed in stress (Shekhar and DiMicco, 1987; Wible et al., 1988;

Received July 24, 1995; revised Oct. 16, 1995; accepted Oct. 20, 1995.

This research was supported by USPHS Grant NS 19883. E.H.S.-P. was supported by a fellowship from the American Heart Association. Indiana Affiliatc. We thank S. Michelle Morin and Amy J. Monroe for their assistance.

Correspondence should be addressed to Joseph A. DiMicco, Department of Pharmacology and Toxicology, Indiana Universily School of Medicine, 6,35 Bamhill Drive. Indianapolis, IN 46202.

Copyright $\odot 1996$ Society for Neurosicnce $0270-6474 / 96 / 161173-07 \$ 05.00 / 0$
Soltis and DiMicco, 1991a,b, 1992; DiMicco et al., 1992; Shekhar, 1993; Sheklıar et al., 1993; DeNovellis et al., 1995; Greenwood and DiMicco, 1995). More significantly, previous injection of muscimol, an agonist at the $\mathrm{GABA}_{\mathrm{A}}$ receptor, at similar stercotaxic coordinates abolished air stress-induced tachycardia (Lisa et al., 1989). The latter finding showed clearly that inhibition of neurons in the dorsal hypothalamus could block stress-induced cardiovascular changes in rats and suggested that these particular neurons play a key role in generating the increases in heart rate and arterial pressure associated with acute experimental stress in rals. Huwever, given the proximity of the DMH to the PVN and the relatively large volume of injection $(250 \mu \mathrm{l})$ used in this study (and also because of the failure to include postmortem histology), the specific site of action of muscimol was unclear. In the present study, we have attempted to determine which of the two nuclei represents the relevant site of action for muscimol by injecting this agent in smaller volumes into histologically identified sites in the medial hypothalamic region including the PVN and the DMH.

\section{MATERIALS AND METHODS}

All experiments were performed in male Sprague-Dawley rats $(300-325$ $\mathrm{gm}$ ) that were housed individually and allowed food and water ad libitum. All procedures were approved by the Institutional Animal Care and Use Committee and followed National Institutes of Health guidelines.

Animals were instrumented initially with telemetric implants (TA11PA-C40, Data Sciences, St. Paul, MN) for monitoring heart rate and blood pressure. While the rats were under pentobarbital anesthesia $(50 \mathrm{mg} / \mathrm{kg}$, i.p.), a midline incision was made in the abdomen, and the descending aorta was exposed between the branching of the renal arteries and the bifurcation of the femoral arteries. The descending aorta was temporarily occluded just distal to the renal arteries, and the telemetric catheter was inserted into the aorta. The catheter was secured in place 
Figure 1. Schematic parasagittal section adapted from Paxinos and Watson (1986) illustrating injection sites in chronically instrumented rats. Bilateral injection sites are shown on the same side to emphasize the relative locations of each injection region. Data are organized according to cannula placements based on criteria outlined in text. ( $(\mathbf{\Delta})$ $\mathrm{DMH}(n=5) ;(\bullet)$ intermediate $(n=4)$; (घ) $P V N(n=5)$. $A H$, Anterior hypothalamic area; $D A$, dorsal hypothalamic area; $D M C$, dorsomedial hypothalamic nucleus, compact; $D M D$, dorsomedial hypothalamic nucleus, diffuse; $D o$, dorsal hypothalamic nucleus; $P H$, posterior hypothalamic area; $P V N$, paraventricular hypothalamic nucleus; $V M H$, ventromedial hypothalamic nucleus.

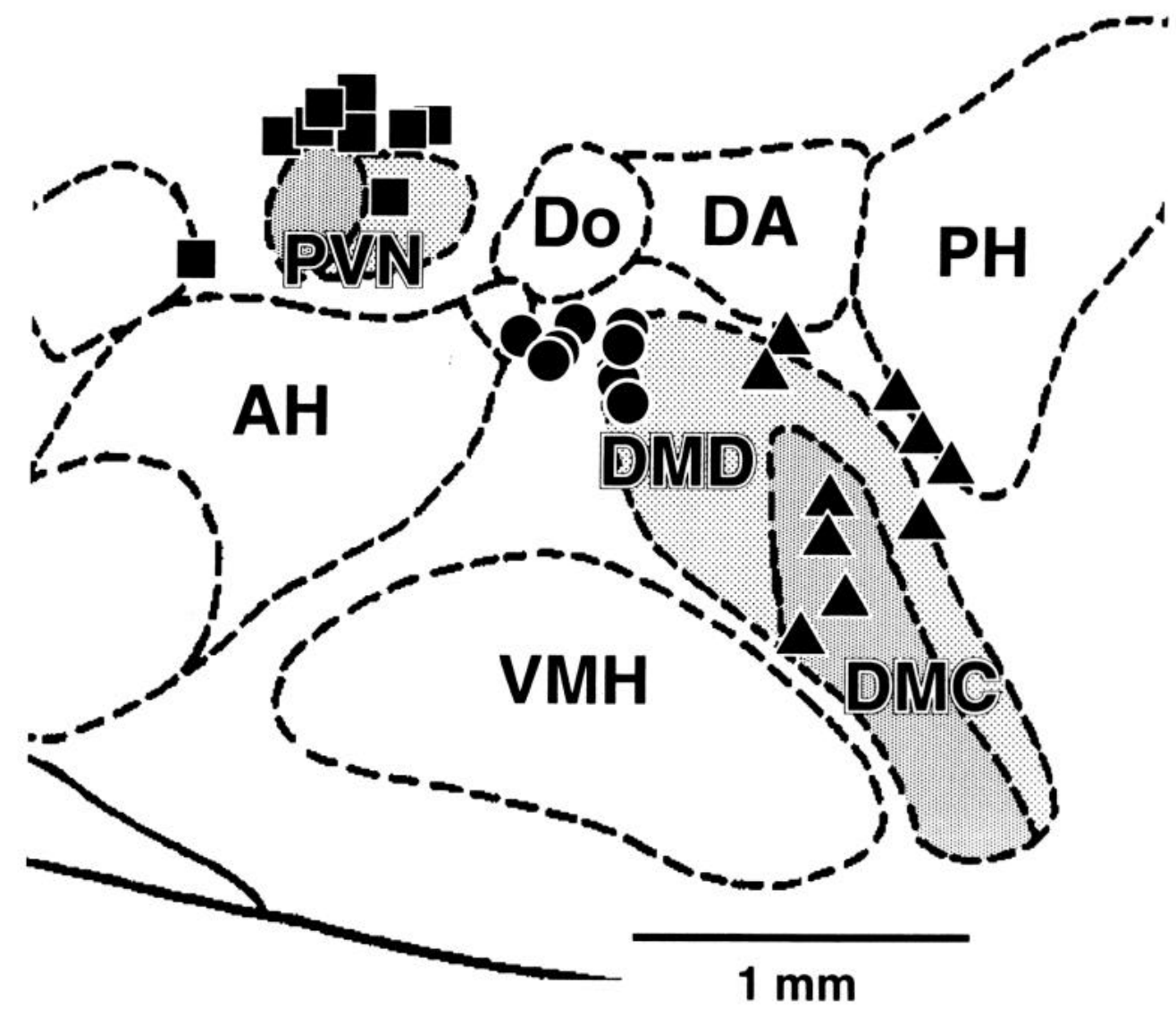

with medical grade tissue adhesive, and the body of the catheter was secured to the muscle wall of the abdomen.

After $7 \mathrm{~d}$ of recovery, rats again were anesthetized with pentobarbital $(30 \mathrm{mg} / \mathrm{kg})$ and placed in a stereotaxic apparatus with an incisor bar $5 \mathrm{~mm}$ above the interaural plane. Two stainless steel guide cannulae (26 gauge, $10 \mathrm{~mm}$ length; Plastics One, Roanoke, VA) were placed bilaterally to allow for injection at stereotaxic coordinates targeted to the PVN, the $\mathrm{DMH}$, or the region between the two nuclei. Coordinates for cannulae placement, using bregma as the reference point and at a $10^{\circ}$ angle from the sagittal, were: (1) DMH, $1.2 \mathrm{~mm}$ posterior, $2.1 \mathrm{~mm}$ lateral, $9.3 \mathrm{~mm}$ ventral; (2) PVN, $0.0 \mathrm{~mm}$ anterior, $2.1 \mathrm{~mm}$ lateral, $8.3 \mathrm{~mm}$ ventral; and (3) intermediate area, $0.6 \mathrm{~mm}$ posterior, $2.1 \mathrm{~mm}$ lateral, $8.8 \mathrm{~mm}$ ventral. Cannulae were secured in place with cranioplastic cement and two stainless steel screws anchored to the skull. Dummy wire cannulae were placed in the guide cannulae, and the rats were returned to their home cage for a $7 \mathrm{~d}$ recovery period.

On the day of testing, the rats were brought to the testing area in their home cages. After $30 \mathrm{~min}$ of baseline heart rate and blood pressure monitoring, either $100 \mathrm{nl}$ of saline or $80 \mathrm{pmol}$ of muscimol in $100 \mathrm{nl}$ saline was injected bilaterally through 33-gauge injectors connected to $10 \mu \mathrm{l}$ syringes mounted on a Harvard infusion pump. Rats then were placed in a restraining tube and subjected to a stream of air directed at the head of the animal at a rate of $40 \mathrm{l} / \mathrm{min}$ as described previously (Lisa et al., 1989). Twenty minutes after the bilateral injections, the air was turned off and the rats were released from the tube. Heart rate and blood pressure were monitored for an additional $20 \mathrm{~min}$ for all but two rats. Each rat was tested twice in the air stress paradigm on alternate days (i.e., days 1 and 3 ), once after injection of saline vehicle and once after injection of muscimol, in staggered order.

Repeated microinjections were found to produce increasing degrees of local tissue damage and cavitation. Consequently, although no effect of previous injections on either stress-induced cardiovascular changes or responsiveness to muscimol (see below) was apparent, precise localization of injection sites often was difficult or impossible. Therefore, in many rats only the two injections described in the protocol above were performed. On the intervening day between stress trials, however, some animals received an additional bilateral injection of muscimol $(80 \mathrm{pmol} /$ $100 \mathrm{nl}$ ) into either the PVN or the DMH to examine the effect on heart rate and arterial pressure under baseline conditions, i.e., without air stress.

At the completion of the study, each rat was anesthetized deeply with pentobarbital, and the injection sites were marked by injection of Alcian blue dye. The rats were perfused transcardially with $60 \mathrm{ml}$ of heparinized saline followed by $120 \mathrm{ml}$ of $10 \%$ buffered formalin, and the brains were removed and stored in $10 \%$ buffered formalin. One to two weeks later, brains were blocked and $40 \mu \mathrm{m}$ sections were cut on a freezing microtome, mounted on slides, and stained with neutral red. The location of the injection sites was determined according to the atlas of Paxinos and Watson (1986).

Data were organized according to anatomical location of the injection sites and analyzed by ANOVA using a random block design or by $t$ tests. After ANOVA, comparisons between individual means were carried out by Fisher's protected least significant difference (PLSD) test. The criterion for statistical significance was $p<0.05$.

\section{RESULTS}

Only data from experiments in which both placements could be determined positively from postmortem histology and that fell

\begin{tabular}{|c|c|c|c|c|}
\hline & \multicolumn{2}{|c|}{ Heart rate (beats/min) } & \multicolumn{2}{|c|}{$\begin{array}{l}\text { Blood pressure } \\
(\mathrm{mmHg})\end{array}$} \\
\hline & Saline & Muscimol & Saline & Muscimol \\
\hline $\mathrm{DMH}(n=5)$ & $363 \pm 4$ & $353 \pm 12$ & $104 \pm 3$ & $104 \pm 3$ \\
\hline Intermediate $(n=4)$ & $369 \pm 3$ & $379 \pm 10$ & $102 \pm 5$ & $107 \pm 6$ \\
\hline $\mathrm{PVN}(n=5)$ & $336 \pm 5^{a}$ & $335 \pm 7^{a}$ & $100 \pm 2$ & $100 \pm 3$ \\
\hline
\end{tabular}

Data represent mean \pm SEM. Statistical analysis reveals that although baseline blood pressure is not different among the treatment groups, baseline heart rate is lower in the PVN groups than in the DMH or intermediate group.

${ }^{a}$ Statistically different from other groups but not different from each other (ANOVA and Fisher's PLSD test, $p<0.05$ ). 
into one of the following three categories were used for analysis (Fig. 1): PVN, both injection sites within $400 \mu \mathrm{m}$ of the main body of the PVN $(n=5)$; DMH, both injection sites in or within 300 $\mu \mathrm{m}$ of the zona compacta of the DMH $(n=5)$; and an intermediate area, both injection sites lying in the region between the DMH and the PVN (including the rostral-most edge of the difluse zone of the $\mathrm{DMH}$ ) and at least $500 \mu \mathrm{m}$ from either the compact zone of the DMH or the main body of the PVN $(n=4)$. All injections were between 200 and $900 \mu \mathrm{m}$ from the third ventricle. Statistical analysis indicated that the average distance from the injection sites to the third ventricle was not significantly different among the three regions ( $F=2.025, p=0.1531$ ).

Baseline heart rate and blood pressure were stable before the injections. Statistical analyses revealed that although baseline blood pressure was not different between the treatment groups, basal heart rate was slightly but significantly lower in rats with guide cannulae implanted in the PVN compared with that in the other two treatment groups (Table 1). After microinjection of saline vehicle, however, air stress elicited immediate and equiva- lent increases in heart rate and mean arterial pressure that persisted throughout the entire $20 \mathrm{~min}$ period in all three groups (Figs. 2, 3). To avoid inclusion of data representing cardiovascular effects caused only by physical struggling and movement typically observed in the first few minutes of the air stress period, we analyzed arterial pressure and heart rate starting 5 min after microinjection, 3-4 min after the beginning of air stress. Mean stress-induced increases in heart rate and arterial pressure were not statistically different among the three groups (Fig. 3). In all but two experiments, both parameters were followed for an additional 20 min after termination of air stress and returned gradually to baseline values (data not shown).

After microinjection of muscimol into the DMH, the cardiovascular response to air stress was nearly abolished compared with that observed after control injection of saline (Figs. 2, 3). Mean tachycardia during the final $15 \mathrm{~min}$ of the stress period was reduced by $85 \%$ after muscimol compared with control, and blood pressure was reduced by $68 \%$. After microinjection of muscimol into the intermediate area, the cardiovascular changes observed

\begin{tabular}{|c|c|c|c|}
\hline-0 & $\begin{array}{l}\text { Saline - All sites } \\
(n=14)\end{array}$ & ----A'--- & $\begin{array}{l}\text { Muscimol - DMH } \\
(n=5)\end{array}$ \\
\hline$\cdots \cdot \cdots$ & $\begin{array}{l}\text { Muscimol - Intermediate } \\
(n=4)\end{array}$ & $\ldots$ & $\begin{array}{l}\text { Muscimol - PVN } \\
(n=5)\end{array}$ \\
\hline
\end{tabular}
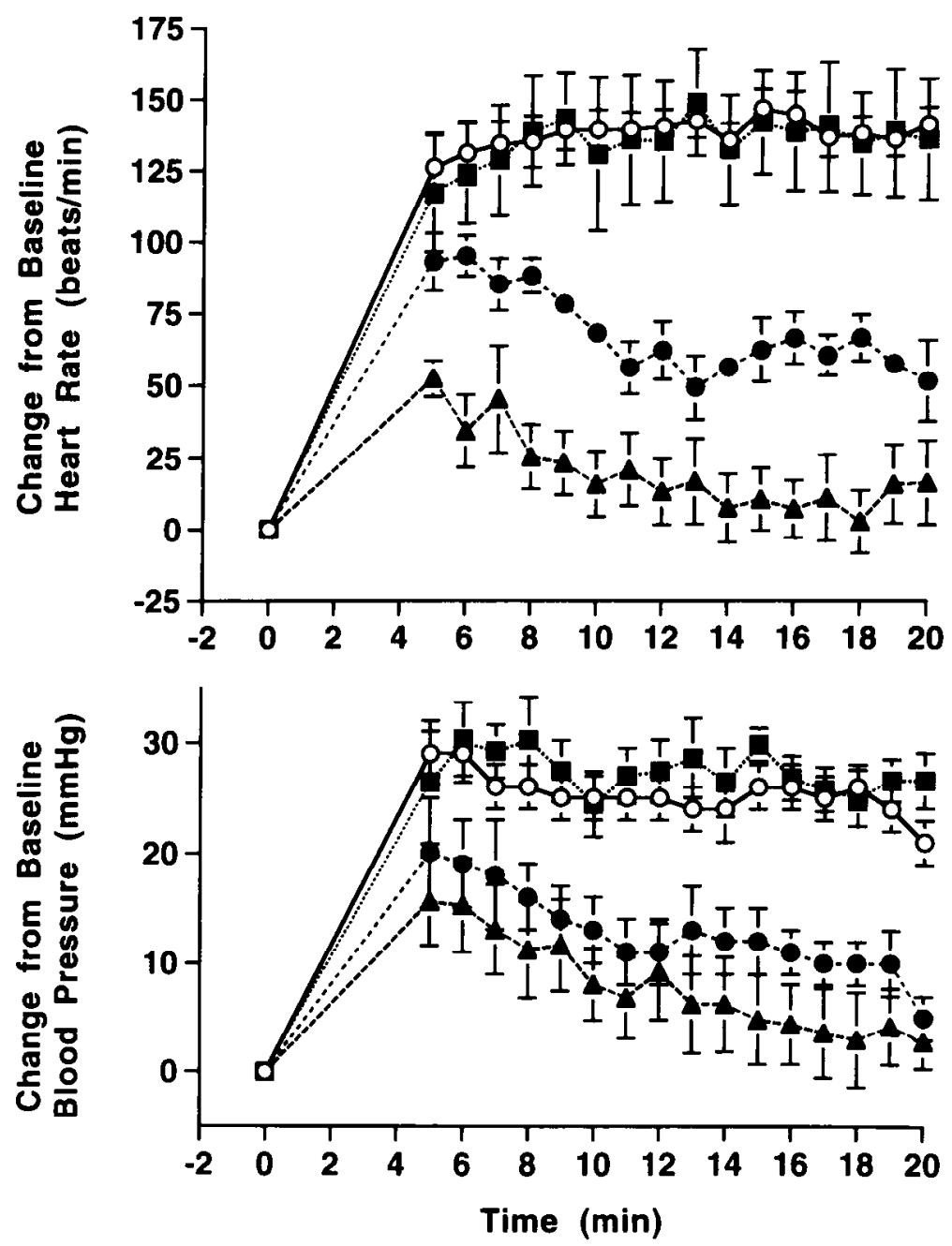

Figure 2. Change from baseline heart rate (top) and blood pressure (bottom) during $20 \mathrm{~min}$ air stress trials after bilateral injection of either vehicle $[100 \mathrm{nl}$ of saline $(\bigcirc)$ or $80 \mathrm{pmol}$ of muscimol] into the DMH $(\mathbf{A})$, intermediate area $(\mathbf{O})$, and PVN (ם). 


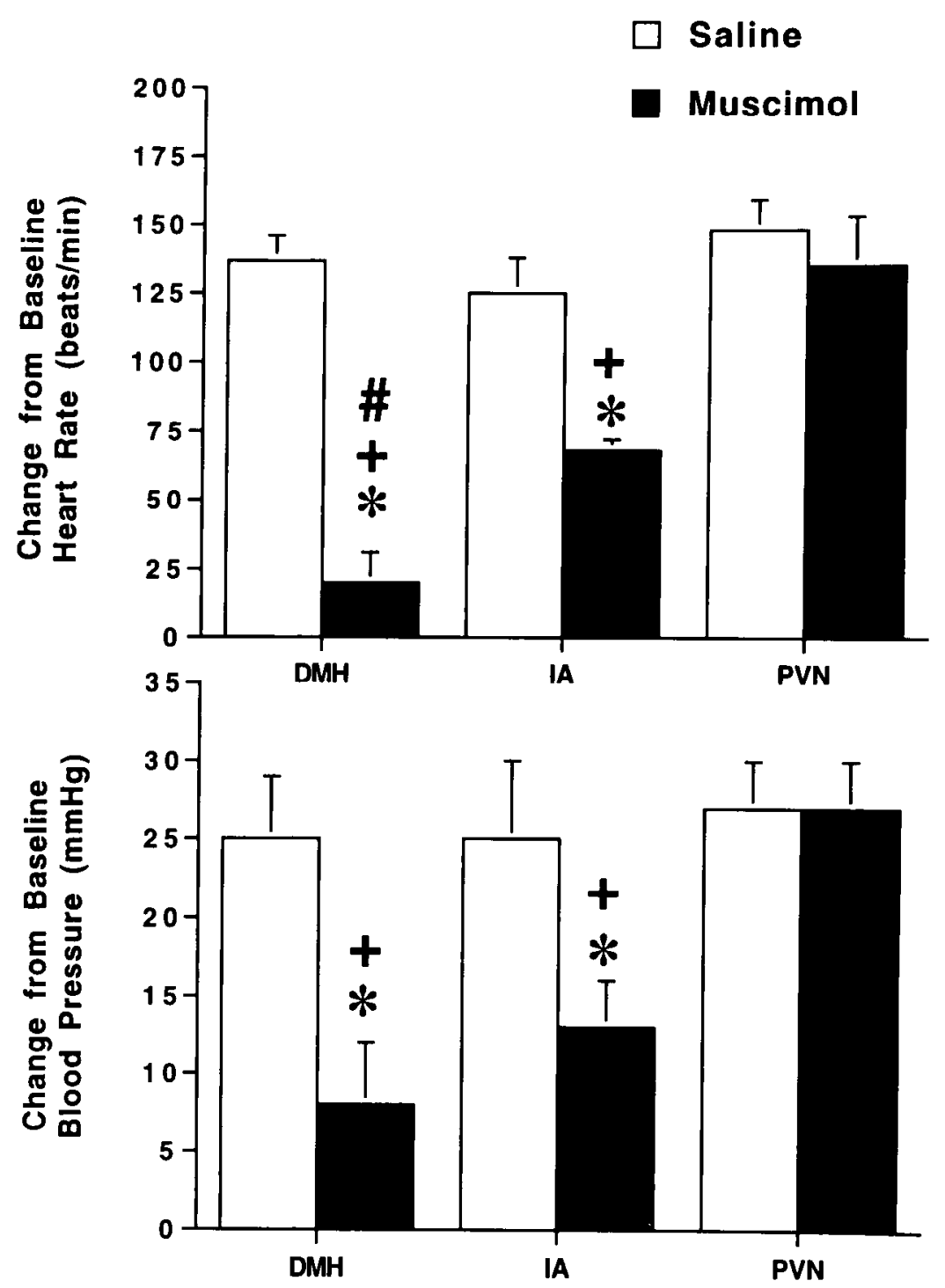

Figure 3. Mean air stress-induced change from baseline heart rate (top) and blood pressure (bottom) after bilateral injections of either vehicle [100 $\mathrm{nl}$ of saline (open hars) or 80 pmol of muscimol (filled bars)] into the region of the zona compacta of the dorsomedial hypothalamic nucleus $(D M H ; n$ $=5$ ), the region of the main body of the paraventricular hypothalamic nucleus $(P V N ; n=5)$, and an area between the two nuclei (intermediate area, $I A ; n=4$ ). Data represent the means from individual trials in which increases over baseline were averaged during the final 15 min of the stress period. "Significantly different from response after injection of saline by paired $t$ test; 'Significantly different from response after injection of muscimol in PVN by two-way ANOVA and Fisher's PLSD test; \#Significantly different from response after injection of muscimol in intermediate area by two-way ANOVA and Fisher's PLSD test.

during air stress also were attenuated compared with those observed after saline (Figs. 2, 3), but to a significantly lesser degree than was observed after identical injection into the DMH. Thus, mean tachycardia and increase in arterial pressure were reduced by 46 and $52 \%$, respectively. In contrast, microinjection of muscimol into the region of the PVN, in which 9 of 10 injection sites were within $300 \mu \mathrm{m}$ of the main body of the nucleus (Fig. 1), failed to influence the cardiovascular response to air stress (Figs. 2, 3).

Two additional animals were found to have bilateral cannula placement posterior to the DMH in the region of the posterior hypothalamus. Injection sites in these experiments were from 300 to $500 \mu \mathrm{m}$ posterior to the zona compacta of the DMH. After injection of saline vehicle in these experiments, air stress evoked cardiovascular changes that were similar to those observed after injection of vehicle in other rats. Microinjection of muscimol at these posterior placements modestly attenuated the cardiovascular response to air stress compared with that observed after control injection of saline. Thus, mean tachycardia during the final $15 \mathrm{~min}$ of the stress period was reduced by $-30 \%$ after muscimol compared with control in both animals, whereas the stress-induced pressor response secmed to be attenuated (by $26 \%$ ) in only one rat.
The relationship between the ability of muscimol to attenuate the cardiovascular response to stress and the site of injection for each individual experiment is indicated in Figure 4. In every experiment in which muscimol was injected within $300 \mu \mathrm{m}$ of the zona compacta of the dorsomedial hypothalamus, the response to air stress was inhibited by at least $50 \%$. However, in experiments in which injection sites were located either anterior or posterior to this region, muscimol was consistently less effective.

In several experiments on the day after the first stress trial, the effects of microinjection of muscimol on cardiovascular function during the $15 \mathrm{~min}$ period analogous to that analyzed in stressed animals also were examined under unstressed conditions. After injection of muscimol into the DMH $(n=4)$, mean differences from baseline heart rate $(+14 \pm 16$ beats $/ \mathrm{min})$ and blood pressure $(+6 \pm 6 \mathrm{mmHg})$ were not significantly different from those observed after injection into the PVN $(+58 \pm 31$ beats $/ \mathrm{min}$ and $+11+5 \mathrm{mmHg}$, respectively; $n=3$ ).

\section{DISCUSSION}

The results of this study clearly indicate that inhibition of neurons with the $\mathrm{GABA}_{\mathrm{A}}$ receptor agonist muscimol in the region of the DMH essentially blocks the increases in heart rate and blood pressure normally observed during air stress. Although similar 


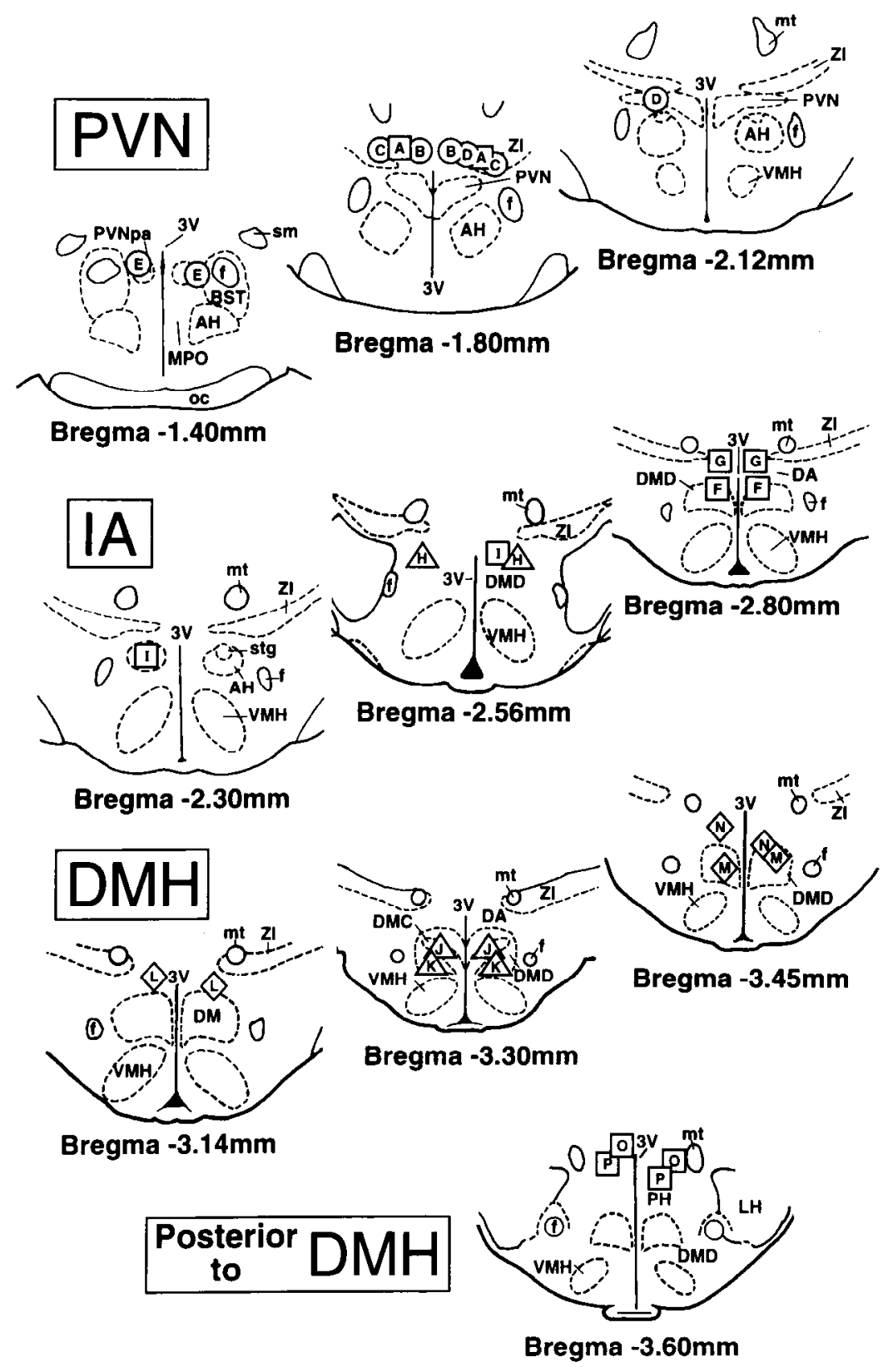

Figure 4. Schematic coronal sections adapted from Paxinos and Watson (1986) illustrating the degree of suppression of air stress-induced tachycardia by muscimol oblained at bilateral injection sites in each chronically instrumented rat. Paired injection sites in individual rats are designated by letters in symbols $(A-P)$. Symbols are coded for the degree of suppression observed in a given animal: (circle) $<25 \%$ suppression: (square) $26-50 \%$ suppression; (triangle) 51-75\% suppression; and (diamond) $>76 \%$ suppression. For the purpose of analyses in Figures 2 and 3 , data from the individual rats are grouped as follows: rats $A-E, P V N(n=5)$; rats $F-I$, intermediate ( $=4)$; rats $J-N, D M H(n=5)$. $A H$, Anterior hypothalamic area; $B S T$, bed nucleus of stria terminalis; $D A$, dorsal hypothalamic area; $D M$, dorsomedial hypothalamic nucleus; $D M C$, dorsomedial hypothalamic nucleus, compact; $D M D$, dorsomedial hypothalamic nucleus, diffuse; $f$, fornix; $L H$, lateral hypothalamus; $M P O$. medial preoptic area; $m t$, mammillothalamic tract; $o c$, optic chiasm; $P H$, posterior hypothalamic area; $P V N$, paraventricular hypothalamic nucleus; $P V N P a$, paraventricular hypothalamic nucleus, parvocellular; $s m$, stria medullaris thalamus; $s t g$, stigmoid hypothalamic nuclcus; $V M H$, ventromedial hypothalamic nucleus; $Z I$, zona incerta; $3 V$, third ventricle. findings were reported previously from our laboratory (Lisa et al., 1989), the precise site of injection was not verified histologically and, in facl, was presumed incorrectly to be the posterior hypothalamus based on the stereotaxic coordinates used and the atlas of Pellegrino et al. (1979). Subsequently, we and others (Spencer et al., 1986) noted this inaccuracy and have established the DMH as the area targeted in earlier and subsequent studies involving chemical stimulation (Anderson and DiMicco, 1990; Soltis and DiMicco, 1991a). In the current experiments, injection sites at which muscimol is most effective in blocking stress-induced cardiovascular effects also were shown to correspond most closely to the DMH.

Other than inclusion of postmortem histology to determine the sites of injection precisely, the most notable difference between this and our previous study was the volume of injection used. Although a larger volume of injection was used previously, microinjection of muscimol bilaterally into the $\mathrm{DMH}$ virtually abolished the cardiovascular response to air stress in both studies. Similar injection of muscimol under baseline conditions had no significant effect on heart rate and arterial pressure in the current study; however, in our earlier report it was followed by modest decreases in both. The apparent discrepancy may have been a consequence of the larger volume of injection allowing more extensive spread of muscimol to other regions, perhaps via the ventricular spaces. In any event, the data suggest that the same neurons in which inhibition markedly reduces stress-induced increases in heart rate and arterial pressure contribute negligibly to autonomic tone to the heart and vasculature under basal conditions.

The most intriguing aspect of the present findings relates to the reduction and then loss of the ability of muscimol to counter stress-induced cardiovascular changes as injection sites were moved away from the DMH anteriorly toward the PVN. Although not included in the statistical analysis, the data from the two rats with guide cannulae placed in the region posterior to the DMH indicate that injection at sites posterior to the DMH also results in a decreased ability of muscimol to block the stress-induced car- 
diovascular changes. Kynurenate, an antagonist at ionotropic excitatory amino acid receptors, attenuated air stress-induced tachycardia when injected into the DMH, but not when injected at the same dose into hypothalamic sites $300-600 \mu \mathrm{m}$ posterior or lateral to this nucleus (Soltis and DiMicco, 1992). This finding suggested that neurons in which activation plays a crucial role in the generation of stress-induced cardiovascular changes were not located posterior or lateral to the $\mathrm{DMH}$, but the involvement of more anterior sites was not examined. The latter point was particularly important because currently prevailing views regard the PVN, located $<1 \mathrm{~mm}$ anterior to the DMH, as the hypothalamic center for integrating autonomic and endocrine responses to stress (Swanson and Sawchenko, 1980). Because muscimol was progressively less effective when injected in identical fashion at sites farther from the DMH and closer to the PVN, the PVN can be ruled out as the site of action for this agent in these studies.

Injection of muscimol into the intermediate area comprising the rostral-most diffuse zone of the DMH and the region between the two nuclei moderately attenuated the cardiovascular response to stress. The most plausible explanation for this effect is that muscimol injected at sites $>500 \mu \mathrm{m}$ from the compact zone of the DMH may diffuse or "spread" to this site to achieve local concentrations capable of eliciting only a partial effect. An alternative interpretation of the data is that the target neurons mediating the effects of injected muscimol may be found primarily in the zona compacta of the DMH and also extending somewhat anteriorly from this region. Thus, microinjection of muscimol into a region anterior to the DMH might produce a reduced effect because fewer of these critical ncurons were inhibited and/or because some or all of these neurons were inhibited to a lesser degree.

Muscimol exerts its inhihitory effect by acting as an agonist at $\mathrm{GABA}_{\mathrm{A}}$ receptors, which virtually all mammalian neurons are thought to possess and by which GABA is thought to be an essentially universal inhibitor of neuronal activity (Brown et al., 1981). In this study, the same dose of muscimol that nearly abolished air stress-induced cardiovascular changes when injected into the DMH had no discernible effect when injected at sites immediately adjacent to the PVN. In fact, in four of the five experiments targeting the PVN, both injection sites were within $300 \mu \mathrm{m}$ of the main body of the PVN. Taken together, these findings clearly preclude the PVN as a potential site of action for microinjected muscimol in this and in our previous study (Lisa et al., 1989). Instead, the results indicate that (1) neurons in the PVN that play a role in generating stress-induced cardiovascular changes, as has been suggested, are much less sensitive to muscimol-induced inhibition than are those in the DMH, so that injection of muscimol at higher doses and/or directly into the PVN might be required to produce discernible effects; or (2) muscimolinduced inhibition of neurons in the PVN has no effect on the generation of the tachycardia and increased blood pressure observed in air stress.

Our findings contrast with the generally accepted concept that neurons in the PVN play a key role in the integration of the autonomically mediated cardiovascular response to stress (Swanson and Sawchenko, 1983; Dampney, 1994). Instead, the reduction of stressinduced tachycardia after lesioning of the PVN reported previously (Callahan et al., 1989) may have been caused by collateral damage to the nearby DMH. Similarly, cardiovascular changes reported after microinjection of the $\mathrm{GABA}_{\wedge}$ receptor antagonist $\mathrm{BMI}$ or kainate into the PVN (Jin and Rockhold, 1989; Martin et al., 1991; Martin and Haywood, 1993) may have becn causcd by spread or diffusion to active sites in the DMH, where microinjection of the same agents at doses orders of magnitude lower elicits similar cardiovascular changes (Soltis and DiMicco, 1991a,b, 1992). In a recent study (DeNovellis et al., 1995), we examined the cardiovascular effects of microinjection of BMI, kainate, and NMDA into the same three regions studied with respect to muscimol here. In parallel with the current findings, each agent evoked a similar pattern of cardiovascular effects, with the most marked increases in heart rate and arterial pressure caused by microinjection into the $\mathrm{DMH}$, little or no effect upon injection into the PVN, and an intermediate response after injection into the region between the two nuclei. These findings suggest that these agents elicit stress-like cardiovascular changes by acting in the same hypothalamic region in which muscimol works to suppress stress-induced increases in heart rate and arterial pressure.

In summary, the data from this study clearly implicate neuronal activity in the DMH in the generation of the cardiovascular changes observed in air stress. However, no evidence was found to support a similar role for neurons in the nearby PVN. These data, taken together with our findings that the active site for excitatory amino acids and BMI is in the DMH rather than in the PVN, suggest that the DMH is a major site for the neuronal control of the cardiovascular response to stress.

\section{REFERENCES}

Anderson JJ, DiMicco JA (1990) Effect of local inhibition of $\gamma$-aminobutyric acid uptake in the dorsomedial hypothalamus on extracellular levels of $\gamma$-aminobutyric acid and on stress-induced tachycardia: a study using microdialysis. J Pharmacol Exp Ther 255:1399-1407.

Brown DA, Higgins AJ, March S, Smart TG (1981) Actions of GABA on mammalian neurones, axons, and nerve terminals. In: Amino acid neurotransmitters (DeFeudis FV, Mandel P, eds), pp 321-326. New York: Raven,

Callahan MF, Kirby RF, Cunningham JT, Eskridge-Sloop SL, Johnson AK, McCarty R, Gruber KA (1989) Central oxylocin systems may mediate a cardiovascular response to acute stress in rats. Am J Physiol 256:H1369-H1377.

Dampney RA (1994) Functional organization of central pathways regulating the cardiovascular system. Physiol Rev 74:323-364.

DeNovellis V, Stotz-Potter EH, Morin SM, Rossi F, DiMicco JA (1995) Hypothalamic sites mediating cardiovascular effects of microinjected bicuculline and $\mathrm{E} \Lambda \Lambda \mathrm{s}$ in rats. Am J Physiol 269:R131-R140.

DiMicco JA, Soltis RP, Anderson JJ, Wible Jr JH (1992) Hypothalamic mechanisms and the cardiovascular response to stress. In: Central neural mechanisms in cardiovascular regulation, Vol 2 (Kunos G, Ciriello J, eds), pp 52-79. Boston: Birkhäuser.

Gelsema AJ, Roe MJ, Calaresu FR (1989) Neuronally mediated cardiovascular responses to stimulation of cell bodies in the hypothalamus of the rat. Brain Res 482:67-77.

Greenwood B, DiMicco JA (1995) Activation of the hypothalamic dorsomedial nucleus stimulates intestinal motility in rats. Am J Physiol 268:G514-G521.

Jin C, Rockhold RW (1989) Effects of paraventricular hypothalamic microinjections of kainic acid on cardiovascular and renal excretory function in conscious rats. J Pharmacol Exp Ther 251:969-975.

Lisa M, Manno E, Wible Jr JH, DiMicco JA (1989) Injection of muscimol into posterior hypothalamus blocks stress-induced tachycardia. Am J Physiol 257:R246-R251.

Martin DS, Haywood JR (1993) Hemodynamic responses to paraventricular nucleus disinhibition with bicuculline in conscious rats. Am J Physiol 265:H1727-H1733.

Martin DS, Haywood JR, Thornhill JA (1993) Stimulation of the hypothalamic paraventricular nucleus causes systemic venoconstriction. Brain Res 604:318-324.

Martin DS, Segura T, Haywood JR (1991) Cardiovascular responses to bicuculline in the paraventricular nucleus of the rat. Hypertension $18: 48-55$.

Paxinos G, Watson C (1986) The rat brain in stereotaxic coordinates, 2nd Ed. New York: Academic.

Pellegrino LJ, Pellegrino AS, Cushman JA (1979) Stereotaxic atlas of the rat brain, 2nd Ed. New York: Plenum. 
Shekhar A (1993) GABA receptors in the region of the dorsomedial hypothalamus of rats regulate anxiety in the elevated plus-maze test. I. Behavioral measures. Brain Res 627:9-16.

Shekhar A, DiMicco JA (1987) Defense reaction elicited by injections of GABA antagonists and synthesis inhibitors into the posterior hypothalamus in rats. $\mathbf{J}$ Neuropharmacol 26:407-417.

Shekhar A, Sims LS, Bowsher RR (1993) GABA receptors in the region of the dorsomedial hypothalamus of rats regulate anxiety in the elevated plus-maze test. II. Physiological measures. Brain Res 627:17-24.

Soltis RP, DiMicco JA (1991a) GABA and excitatory amino acid receptors in dorsomedial hypothalamus and heart rate in rats. Am J Physiol 260:R13-R20.

Soltis RP, DiMicco JA (1991b) Interaction of hypothalamic GABA $\mathrm{A}_{\mathrm{A}}$ and excitatory amino acid receptors controlling heart rate in rats. Am $\mathbf{J}$ Physiol 261:R427-R433.
Soltis RP, DiMicco JA (1992) Hypothalamic excitatory amino acid receptors mediate stress-induced tachycardia in rats. Am J Physio 262:R689-R697.

Spencer SE, Sawyer WB, Loewy AD (1986) L-Glutamate mapping of cardioreactive areas in the rat posterior hypothalamus. Brain Res Bull $16: 231-248$.

Swanson LW, Sawchenko PE (1980) Paraventricular nucleus: a site for the integration of neuroendocrine and autonomic mechanisms. Neuroendocrinology 31:410-417.

Swanson LW, Sawchenko PE (1983) Hypothalamic integration: organization of the paraventricular and supraoptic nuclei. Annu Rev Neurosci $6: 269-324$.

Wible Jr JII, Luft FC, DiMicco JA (1988) Ilypothalamic GABA suppresses sympathetic outflow to the cardiovascular system. Am J Physiol 254:R680-R687. 